\title{
An Omnibus Test for Univariate and Multivariate Normality
}

\author{
By JURGEN A. DOORNIK ${ }^{\dagger}$ \\ and \\ HENRIK HANSEN \\ Nuffield College, Oxford \\ University of Copenhagen, Denmark
}

November 24, 1994

\section{SUMMARY}

We suggest an easy to use version of the omnibus test for normality using skewness and kurtosis based on Shenton and Bowman (1977) which controls well for size. A multivariate version is introduced. Size and power are investigated in comparison with four other tests for multivariate normality. The alternative hypothesis in the power simulations is the whole Johnson system of distributions.

Keywords: JOHNSON SYSTEM; KURTOSIS; MULTIVARIATE NORMALITY TEST; SKEWNESS; UNIVARIATE NORM ALITY TEST; WILSON-HILFERTY TRANSFORMATION

\section{INTRODUCTION}

Testing for normality is a common procedure in much applied work and many tests have been proposed, see for example the overviews in Mardia (1980), D'Agostino (1982) and Small (1985). The need for testing normality in a multivariate setting is discussed, inter alia, by Gnanadesikan (1977, §5.4.2), Cox and Small (1978) and recently Cox and Wermuth (1994).

A test frequently used is the sum of squares of the standardized sample skewness and kurtosis, which is asymptotically distributed as a $\chi^{2}$-variate. For small samples the transformation to approximate $\chi^{2}$ as given in Bowman and Shenton (1975) is cumbersome, and many computer programs only report the asymptotic version of the test.

The next section bases a more convenient test statistic on work by Shenton and Bowman (1977) (a possibility already mentioned by Pearson, D'Agostino and Bowman, 1977), who give the kurtosis a conditional gamma distribution. The test statistic uses transformed skewness and kurtosis, and is simple to implement. A multivariate version of the test is introduced in $\S 3$. Size and power comparisons with several other multivariate tests are given in $\S 5$ and $\S 6$.

All results in this paper are based on random samples. In practice, however, the tests will also be applied to regression residuals and residuals from time series models.

\footnotetext{
$\dagger$ Address for correspondence: Nuffield College, Oxford OX1 1NF, UK
} 
Limited numerical results in Pierce and Gray (1982), Pierce (1985) and Lütkepohl and Schneider (1989) suggest that the size of the tests is largely unaffected, but there could be some loss of power.

\section{THE UNIVARIATE OMNIBUS TEST}

Let $\left(x_{1}, \ldots, x_{n}\right)$ be a sample of $n$ independent observations on a 1-dimensional random variable with mean $\mu$ and variance $\sigma^{2}$. Write $\mu_{i}=E(X-\mu)^{i}$, so that $\sigma^{2}=\mu_{2}$. The skewness and kurtosis are defined as:

$$
\sqrt{ } \beta_{1}=\frac{\mu_{3}}{\mu_{2}^{3 / 2}} \text { and } \beta_{2}=\frac{\mu_{4}}{\mu_{2}^{2}} .
$$

Sample counterparts are defined by:

$$
\bar{x}=\frac{1}{n} \sum_{i=1}^{n} x_{i}, m_{i}=\frac{1}{n} \sum_{i=1}^{n}\left(x_{i}-\bar{x}\right)^{i}, \sqrt{ } b_{1}=\frac{m_{3}}{m_{2}^{3 / 2}} \text { and } b_{2}=\frac{m_{4}}{m_{2}^{2}} .
$$

Bowman and Shenton (1975) consider the test ( $\widetilde{a}$ denotes 'asymptotically distributed as'):

$$
E_{p}^{a}=\frac{n\left(\sqrt{ } b_{1}\right)^{2}}{6}+\frac{n\left(b_{2}-3\right)^{2}}{24} \widetilde{a} \chi^{2}(2)
$$

unsuitable except in very large samples. The statistics $\sqrt{ } b_{1}$ and $b_{2}$ are not independently distributed (although uncorrelated), and the sample kurtosis especially approaches normality very slowly. They proceed to derive a test based on approximating the distribution of $\sqrt{ } b_{1}$ and $b_{2}$ by the Johnson system (see e.g. Kendall, Stuart and Ord, 1987, $\S 6.27-36$, or Pearson and Hartley, 1972, §18), assuming independence. The Johnson $S_{U}$ approximation to $\sqrt{ } b_{1}$ is given in D'Agostino (1970). The kurtosis part is less convenient, as it involves iteration to find the parameters of the fitting distribution (owing to a Johnson $S_{B}$ being used for $\left.n<25\right)$.

The test considered here derives from Shenton and Bowman (1977), who give $b_{2}$ (conditional on $b_{2}>1+b_{1}$ ) a gamma distribution. The distribution of $\sqrt{ } b_{1}$ is still based on Johnson $S_{U}$. Let $z_{1}$ and $z_{2}$ denote the transformed skewness and kurtosis, where the transformation creates statistics which are much closer to standard normal. The test statistic is ( $\widehat{a p p}$ denotes 'approximately distributed as'):

$$
E_{p}=z_{1}^{2}+z_{2}^{2} \widehat{a p p} \chi^{2}(2) .
$$

The transformation for the skewness is as in D'Agostino (1970); the kurtosis is transformed from a gamma distribution to $\chi^{2}$, which is then translated into standard normal using the Wilson-Hilferty cubed root transformation. The precise form of $z_{1}$ and $z_{2}$ is given in the appendix, together with a numerical example. The advantage of this statistic is that is easy to implement and requires only tables of the $\chi^{2}$ distribution. Note that the formulae break down for $n \leq 7$. 


\section{THE MULTIVARIATE OMNIBUS TEST}

Let $X^{\prime}=\left(X_{1}, \ldots, X_{n}\right)$ be a $p \times n$ matrix of $n$ observations on a $p$-dimensional vector with sample mean and covariance $\bar{X}=n^{-1}\left(X_{1}+\ldots+X_{n}\right)$ and $S=n^{-1} \check{X}^{\prime} \check{X}$ where $\check{X}^{\prime}=\left(X_{1}-\bar{X}, \ldots, X_{n}-\bar{X}\right)$.

Create a matrix with the reciprocals of the standard deviation on the diagonal:

$$
V=\operatorname{diag}\left(S_{11}^{-1 / 2}, \ldots, S_{p p}^{-1 / 2}\right)
$$

and form the correlation matrix $C=V S V$. Define the $p \times n$ matrix of transformed observations:

$$
R^{\prime}=H \Lambda^{-1 / 2} H^{\prime} V \check{X}^{\prime}
$$

with $\Lambda=\operatorname{diag}\left(\lambda_{1}, \ldots, \lambda_{n}\right)$, the matrix with the eigenvalues of $C$ on the diagonal. The columns of $H$ are the corresponding eigenvectors, such that $H^{\prime} H=I_{p}$ and $\Lambda=H^{\prime} C H$. Using population values for $C$ and $V$, a multivariate normal can thus be transformed into independent standard normals; using sample values this is only approximately so.

We may now compute univariate skewness and kurtosis of each transformed $n$-vector of observations. Defining $B_{1}^{\prime}=\left(\sqrt{ } b_{11}, \ldots, \sqrt{ } b_{1 p}\right), B_{2}^{\prime}=\left(b_{21}, \ldots, b_{2 p}\right)$ and $\iota$ as a $p$-vector of ones, the test statistic:

$$
\frac{n B_{1}^{\prime} B_{1}}{6}+\frac{n\left(B_{2}-3 \iota\right)^{\prime}\left(B_{2}-3 \iota\right)}{24} \widetilde{a} \chi^{2}(2 p)
$$

will again require large samples.

The proposed multivariate statistic is:

$$
E_{p}=Z_{1}^{\prime} Z_{1}+Z_{2}^{\prime} Z_{2} \widehat{a p p} \chi^{2}(2 p),
$$

where $Z_{1}^{\prime}=\left(z_{11}, \ldots, z_{1 p}\right)$ and $Z_{2}^{\prime}=\left(z_{21}, \ldots, z_{2 p}\right)$ are determined by (1) and (2) given in the appendix. So after transformation of the data to approximately independent standard normals, the univariate test is applied to each dimension. Using the correlation matrix, rather than the covariance, makes the test scale invariant. Basing the square root of $C$ on the eigenvectors gives invariance to ordering (using Choleski decomposition to compute the square root is inadvisable, as it makes the test sensitive to the ordering of the variables). In practice it will be useful to inspect all elements in $Z_{1}$ and $Z_{2}$ and maybe even compute the transformed skewness and kurtosis of the original data vectors.

If the rank of $C$ is less than $p$, some eigenvalues will be zero. In that case the following procedure can be used. Select the eigenvectors corresponding to the $p^{*}$ nonzero eigenvalues, $G$ say, and create a new data matrix $X^{*}=X G$. This will be an $n \times p^{*}$ matrix. Now compute $E_{p}$ using $X^{*}$ and base the tests on $p^{*}$ degrees of freedom. Simulations showed that this works satisfactorily.

\section{FOUR OTHER MULTIVARIATE TESTS}

Four other tests for multivariate normality are used in our size and power comparisons. Whereas $\S 3$ suggests to first transform to approximate independent normality and then 
compute marginal skewness and kurtosis coefficients, Small (1980) took the opposite route: weight the marginal skewness and kurtosis coefficients of the raw variables by their approximate correlations. Let $B_{1}$ and $B_{2}$ denote the vectors of sample skewness and kurtosis of the original data, which has sample correlation matrix $C=\left(c_{i j}\right)$. Small (1980) used the Bowman and Shenton (1975) correction, but here we use $\S 2$ to compute the transformed skewness and kurtosis of the original data vectors obtaining $Z_{1}$ and $Z_{2}$, and:

$$
Q_{p}=Z_{1}^{\prime} U_{1}^{-1} Z_{1}+Z_{2}^{\prime} U_{2}^{-1} Z_{2} \underset{\text { app }}{ } \chi^{2}(2 p),
$$

where $U_{1}=\left(c_{i j}^{3}\right)$ and $U_{2}=\left(c_{i j}^{4}\right)$.

The next test is that proposed by Mardia (1970). Create the $n \times n$ matrix:

$$
D=\left(d_{i j}\right)=\check{X} S^{-1} \check{X}^{\prime},
$$

and define multivariate measures of skewness and kurtosis as:

$$
b_{1 p}^{*}=\frac{1}{n^{2}} \sum_{i=1}^{n} \sum_{j=1}^{n} d_{i j}^{3} \text { and } b_{2 p}^{*}=\frac{1}{n} \sum_{i=1}^{n} d_{i i}^{4} .
$$

An omnibus test based on these measures is:

$$
M_{p}=\frac{n b_{1 p}^{*}}{6}+\frac{n\left(b_{2 p}^{*}-p(p+2)\right)^{2}}{8 p(p+2)} \widetilde{a} \chi^{2}\left(\frac{p(p+1)(p+2)}{6}+1\right) .
$$

A large sample will be required for the $\chi^{2}$ distribution to hold.

Recently, Mudholkar, McDermott and Srivastava (1992) proposed a new test of multivariate normality based on the correlation between the normalized diagonal of $D$ and jackknifed estimates of its variance. At various stages the statistic is transformed and empirically adjusted, resulting in a statistic which is approximately normally distributed. We square this statistic to obtain the $\chi^{2}$ equivalent:

$$
Z_{p}=\left(\frac{z_{p}+A_{1}(p) / n-A_{2}(p) / n^{2}}{\left\{B_{1}(p) / n-B_{2}(p) / n^{2}\right\}^{\frac{1}{2}}}\right)^{2} \widetilde{a p p} \chi^{2}(1) .
$$

The original paper shows how to obtain $A_{i}$ and $B_{i}$. To obtain a correct size for the test statistic, we found that we had to add $\mu_{n, p}=A_{1}(p) / n-A_{2}(p) / n^{2}$ to $z_{p}$ rather than subtract it.

Finally, we consider the multivariate Shapiro-Wilk test as proposed by Royston (1983). This combines the univariate tests (see Royston, 1982) creating a statistic $H$ which has an approximate $\chi^{2}$ distribution with non-integer degrees of freedom $e$, where $1 \leq e \leq p$. For convenience we translate this into a $\chi^{2}(1)$ using the Wilson-Hilferty approximation:

$$
w=\left\{\left(\frac{H}{e}\right)^{\frac{1}{3}}-1+\frac{2}{9 e}\right\}\left(\frac{9 e}{2}\right)^{\frac{1}{2}}, W_{p}=w^{2} \widehat{a p p} \chi^{2}(1) .
$$




\section{SIZE OF THE TESTS}

To investigate the size of the $E_{p}$ test we simulated rejection frequencies under the null of normality, using $\chi^{2}(2 p)$ critical values. The reported simulations were done in $\mathrm{Ox}$ (a $\mathrm{C}++$ like matrix language), using the internal random number generator. The computations were repeated in Gauss, resulting in minor descrepancies owing to a different random number generator. Each table is based on common random numbers. We report the approximate Monte Carlo standard error as MCSE $=\sqrt{ }\{q(1-q) / M\}$ where $M$ is the number of replications and $q$ the tail probability of interest.

Table 1 contrasts $E_{p}$ with its asymptotic version $\left(E_{p}^{a}\right)$ and the form suggested by Anscombe and Glynn (1983), labelled $E_{p}^{V}$. The latter uses the Pearson type V distribution for the kurtosis and a Johnson $S_{U}$ for the skewness. Table 1 shows that this does not control the size as well as $E_{p}$. Figure 1 illustrates the benefits of taking the dependence into account.

Tables 2 and 3 give the size for some multivariate cases. In Table 2 the simulations are under the null of independent normality, with all tests showing acceptable size, although $W_{p}$ shows that the Wilson-Hilferty approximation which we adopted only works moderately well in the bivariate case. Table 3 is for the bivariate normal case, with various correlation coefficients. It shows that $Q_{p}$ is more sensitive to $\rho$ than $E_{p}$. Both $M_{p}$ and $Z_{p}$ are invariant to $\rho$.

\section{POWER COMPARISONS}

For the power simulations we compute rejection frequencies at $5 \%$ under the alternative distribution, using a sample size of 50 observations. The critical values for $E_{p}, Z_{p}$ and $W_{p}$ are taken from the $\chi^{2}$ table; the $5 \%$ critical values for $M_{p}$ are found by simulation: 18.2 for $p=3, n=50$ and 10.0 for $p=2, n=50$.

In the first set of experiments we use the entire Johnson system as the set of alternative distributions. This results in power simulations forming a two-dimensional grid over the skewness-kurtosis plane. A similar approach was adopted by Baringhaus, Danschke and Henze (1989) who considered four univariate tests, one of them the test of Bowman and Shenton (1975). Because of symmetry we only consider positive skewness. Remember that $\beta_{2}>1+\beta_{1}$ always; power in that part of the skewness-kurtosis plane which does not satisfy this relation are set to zero, resulting in the flat area in the far corner. Along the edge $\beta_{2}=1+\beta_{1}$ are the bimodal distributions. The normal distribution is at $\left(\sqrt{ } \beta_{1}, \beta_{2}\right)=(0,3)$; the exponential at $(2,9)$, just outside the graphs.

The experiments use three independent random variables, and can be summarized as follows:

$\begin{array}{ccccccc}\text { label } & n & M & p & X_{1} & X_{2} & X_{3} \\ \text { P12 } & 50 & 1000 & 3 & \text { Johnson } & \text { standard normal } & \text { standard normal } \\ \text { P30 } & 50 & 1000 & 3 & \text { Johnson } & \text { Johnson } & \text { Johnson }\end{array}$

The graphs are presented for P12 only, for the test statistics $E_{p}, Z_{p}$ and $M_{p} ; \mathrm{P} 12$ for $Q_{p}$ very closely resembles that of $E_{p}$ and is omitted. We see from Figs. $2-4$ that $E_{p}$ has 
the best power properties. $Z_{p}$ has virtually no power against any of these alternatives. $Z_{p}$ focuses only on the elements of $D$ which form the kurtosis component of $M_{p}$, so low power against skewed alternatives is expected, however, power in the kurtosis dimension is absent too. Figure 5 shows the difference of power between $E_{p}$ and $W_{p}$, and confirms an established result for the univariate case, namely that the Shapiro-Wilk test has higher power against platykurtic distributions, and lower power against leptokurtic distributions. Or more precisely: Shapiro-Wilk has higher power against alternatives in the bimodal area of the Johnson $S_{B}$ system, but lower against $S_{U}$. The graphs for the P30 simulations are not presented, but give the same overall picture: all powers have improved, but the relative ranking remains the same.

We experimented with an alternative form of the proposed test statistic $E_{p}$ using only one degree of freedom for the kurtosis component: the $p \chi^{2}$ components of the kurtosis are added up, giving (non-integer) degrees of freedom $\alpha=p a+c \sum b_{1 i}$ for $\chi=2 k \sum\left(b_{2 i}-1-b_{1 i}\right)$, to which the Wilson-Hilferty transformation is applied (these replace the $\alpha$ and $\chi$ in (2), given in the appendix). This alternative test results in somewhat better size properties, but a loss of power in low kurtosis directions (a property shared by $M_{p}$ which also has only one kurtosis component). This form could be useful if all kurtosis components show departure in the same direction.

In the next set of power experiments, the alternative is the generalized Burr-Paretologistic distribution with normal marginals, see Cook and Johnson (1986). The parameters $\alpha$ and $\beta$ correspond to the notation used there. The case of $\alpha \rightarrow \infty$ and $\beta=0$ corresponds to independent normals, whereas $\alpha \rightarrow \infty$ for $\beta \neq 0$ is Morgenstern's bivariate uniform distribution, still with normal marginals. We see in Table 4 that Mardia's test performs best here, followed by $E_{p}$. The most salient feature, however, is the complete lack of power of the $Q_{p}$ and $W_{p}$ tests against a non-normal bivariate distribution with normal marginals. It is interesting to note that the test suggested by Malkovich and Afifi (1973), which consists of that linear combination of the variables which maximizes skewness or kurtosis, will also fail in this situation. The $W_{p}^{*}$ statistic is an alternative form of the multivariate Shapiro-Wilk test, where the data are first transformed to approximate independent normality as for $E_{p}$; then the $p$ univariate Shapiro-Wilk tests are squared and added up, resulting in a $\chi^{2}(p)$ test statistic.

We conclude that $E_{p}$ is the preferred test of the four considered. It is both simple, has correct size and good power properties. However, $E_{p}$ could potentially be used in conjunction with $M_{p}$.

\section{ACKNOWLEDGEMENTS}

We wish to thank Sir David Cox, David Hendry, Chris Orme and Neil Shephard for helpful comments. Financial support from the UK Economic and Social Science Research Council is gratefully acknowledged by Jurgen Doornik. 


\section{APPENDIX}

The transformation for the skewness $\sqrt{ } b_{1}$ into $z_{1}$ is as in D'Agostino (1970):

$$
\begin{aligned}
\beta & =\frac{3\left(n^{2}+27 n-70\right)(n+1)(n+3)}{(n-2)(n+5)(n+7)(n+9)}, \\
\omega^{2} & =-1+\{2(\beta-1)\}^{\frac{1}{2}} \\
\delta & =\frac{1}{\left\{\log \left(\sqrt{ } \omega^{2}\right)\right\}^{\frac{1}{2}}}, \\
y & =\sqrt{ } b_{1}\left\{\frac{\omega^{2}-1}{2} \frac{(n+1)(n+3)}{6(n-2)}\right\}^{\frac{1}{2}} \\
z_{1} & =\delta \log \left\{y+\left(y^{2}+1\right)^{\frac{1}{2}}\right\} .
\end{aligned}
$$

The kurtosis $b_{2}$ is transformed from a gamma distribution to $\chi^{2}$, which is then translated into standard normal $z_{2}$ using the Wilson-Hilferty cubed root transformation:

$$
\begin{aligned}
\delta & =(n-3)(n+1)\left(n^{2}+15 n-4\right) \\
a & =\frac{(n-2)(n+5)(n+7)\left(n^{2}+27 n-70\right)}{6 \delta} \\
c & =\frac{(n-7)(n+5)(n+7)\left(n^{2}+2 n-5\right)}{6 \delta} \\
k & =\frac{(n+5)(n+7)\left(n^{3}+37 n^{2}+11 n-313\right)}{12 \delta} \\
\alpha & =a+b_{1} c, \\
\chi & =\left(b_{2}-1-b_{1}\right) 2 k \\
z_{2} & =\left\{\left(\frac{\chi}{2 \alpha}\right)^{\frac{1}{3}}-1+\frac{1}{9 \alpha}\right\}(9 \alpha)^{\frac{1}{2}}
\end{aligned}
$$

We base a numerical example on the iris data of Fisher (1936), which was used for the same purpose by Small (1980) and Fang and Wang (1994, example 6.5). Using the 50 observations for Iris Setosa on sepal length, sepal width, petal length and petal width, we find for each individual variable: skewness $(0.11645,0.039921,0.10318,1.2159)$ and kurtosis $(2.6542,3.7442,3.8046,4.4343)$. Applying the transformation to approximate normality discussed in $\S 3$ yields skewness $B_{1}^{\prime}=(0.19965,-0.17132,0.15837,1.1610)$ and kurtosis $B_{2}^{\prime}=(2.8221,4.1994,3.9722,4.5793)$. Application of (1) and (2) then yields $Z_{1}^{\prime}=(0.63839,-0.54876,0.50762,3.1862)$ and $Z_{2}^{\prime}=(0.24687,2.5423,2.2381,-1.3278)$. We see that after rotation, skewness is a problem in one dimension, whereas excess kurtosis is evident in two dimensions. The resulting test statistics are: $E_{p}=24.4145$, $Q_{p}=24.0387, M_{p}=27.3413, Z_{p}=1.12299$. Both $E_{p}$ and $Q_{p}$ are significant at less than $1 \%$, whereas the $Z_{p}$ value corresponds to the 29 per cent point, and $M_{p}$ to $10 \%$ (based on simulation results). In this case the non-normality of petal width seems to be the main factor, as without that variable non-normality is not rejected at the $5 \%$ level, with test-statistics of $10.8174(9 \%), 8.74419(19 \%), 12.9066(20 \%)$ and $1.06251(30 \%)$ respectively. The same conclusion was reached by Royston (1983). 


\section{REFERENCES}

Anscombe, G. J. and Glynn, D. F. (1983). Distribution of the kurtosis statistic $b_{2}$ for normal samples. Biometrika 70, 227-234.

Baringhaus, L., Danschke, R. and Henze, N. (1989). Recent and classical tests for normality - A comparative study. Comm. Statist. B 18, 363-379.

Bowman, K. O. and Shenton, L. R. (1975). Omnibus test contours for departures from normality based on $\sqrt{ } b_{1}$ and $b_{2}$. Biometrika $62,243-250$.

Cook, R. D. and Johnson, M. E. (1986). Generalized Burr-Pareto-logistic distributions with applications to a uranium exploration data set. Technometrics 28, 123-131.

Cox, D. R. and Small, N. J. H. (1978). Testing multivariate normality. Biometrika 65, $263-272$.

Cox, D. R. and Wermuth, N. (1994). Tests of linearity, multivariate normality and the adequacy of linear scores. Appl. Statist. 43, 347-355.

D'Agostino, R. B. (1970). Transformation to normality of the null distribution of $g_{1}$. Biometrika 57, 679-681.

— (1982). Departures from normality, testing for. In Kotz, S., Johnson, N. L. and Read, C. B. (eds.), Encyclopedia of Statistical Sciences, vol. 2. Amsterdam: NorthHolland.

Fang, K.-T. and Wang, Y. (1994). Number-theoretic Methods in Statistics. London: Chapman and Hall.

Fisher, R. A. (1936). The use of multiple measurements in taxonomic problems. Annals of Eugenics, 7, 179-188. Reprinted in R.A. Fisher (1950). Contributions to Mathematical Sciences. London: John Wiley and Sons.

Gnanadesikan, R. (1977). Methods for Statistical Data Analysis of Multivariate Observations. London: John Wiley and Sons.

Kendall, M. G., Stuart, A. and Ord, J. K. (1987). Advanced Theory of Statistics 5th edn., vol. 1. London: Charles Griffin and Co.

Lütkepohl, H. and Schneider, W. (1989). Testing for normality of autoregressive time series. Comp. Statist. Quart. 2, 151-168.

Malkovich, J. F. and Afifi, A. A. (1973). Testing for normality of autoregressive time series. J. Am. Statist. Ass. 68, 176-179.

Mardia, K. V. (1970). Measures of multivariate skewness and kurtosis. Biometrika 57, $519-530$.

— (1980). Tests of univariate and multivariate normality. In Krishnaiah, P. R. (ed.), Handbook of Statistics, vol. 1, ch. 9. Amsterdam: North-Holland.

Mudholkar, G. S., McDermott, M. and Srivastava, D. K. (1992). A test of $p$-variate normality. Biometrika 79, 850-854.

Pearson, E. S., D'Agostino, R. B. and Bowman, K. O. (1977). Test for departure from normality: Comparison of powers. Biometrika 64, 231-246. 
Pearson, E. S. and Hartley, H. O. (1972). Biometrika Tables for Statisticians, vol. II. Cambridge: Cambridge University Press.

Pierce, D. A. (1985). Testing normality of errors in regression models. Biometrika $\mathbf{7 2}$, 293-297.

Pierce, D. A. and Gray, R. J. (1982). Testing normality of errors in regression models. Biometrika 69, 233-236.

Royston, J. P. (1982). An extension of Shapiro and Wilk's $W$ test for normality to large samples. Appl. Statist. 31, 115-124.

- (1983). Some techniques for assessing multivariate normality based on the ShapiroWilk W. Appl. Statist. 32, 121-133.

Shenton, L. R. and Bowman, K. O. (1977). A bivariate model for the distribution of $\sqrt{ } b_{1}$ and $b_{2}$. J. Am. Statist. Ass. 72, 206-211.

Small, N. J. H. (1980). Marginal skewness and kurtosis in testing multivariate normality. Appl. Statist. 29, 85-87.

- (1985). Multivariate normality, testing for. In Kotz, S., Johnson, N. L. and Read, C. B. (eds.), Encyclopedia of Statistical Sciences, vol. 6. Amsterdam: NorthHolland. 
Table 1. Empirical size of $E_{p}^{a}, E_{p}^{V}$ and $E_{p}$ for $p=1, M=10000$.

\begin{tabular}{|c|c|c|c|}
\hline & $E_{p}^{a}$ & $E_{p}^{V}$ & 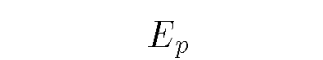 \\
\hline $\mathrm{n}$ & $20 \% \quad 10 \% \quad 5 \% \quad 1 \%$ & $20 \% \quad 10 \% \quad 5 \% \quad 1 \%$ & $20 \% \quad 10 \% \quad 5 \% \quad 1 \%$ \\
\hline 10 & 033.017.010.002. & .024.053.057. & .204 .101 .04 \\
\hline 20 & .062.036.022. 010. & .018. 055. 091. & 011. 046. 092. \\
\hline 50 & .094.055.035.012 & .017. 095. 055. & . 178.088 .046 .011 \\
\hline 50 & .018. 138.070 .044 & .016. 095. 191. & . 182.092 .047 .012 \\
\hline & 002.001 & 001.004. 002. & .004 .003 \\
\hline
\end{tabular}

Table 2. Empirical size of $E_{p}, Q_{p}, Z_{p}$ and $W_{p}$ for $p=2,3,6, M=10000$.

\begin{tabular}{|c|c|c|c|c|}
\hline & $E_{p}$ & $Q_{p}$ & $Z_{p}$ & $W_{p}$ \\
\hline$n, p$ & $20 \% \quad 10 \% \quad 5 \% \quad 1 \%$ & $20 \% \quad 10 \% \quad 5 \% \quad 1 \%$ & $20 \% \quad 10 \% \quad 5 \% \quad 1 \%$ & $20 \% \quad 10 \% \quad 5 \% \quad 1 \%$ \\
\hline 20,2 & 011. 049. 097. 184. & .009.046. 097. 183. & .225. 110.051.007 & 500.038. 089. 190. \\
\hline 50,2 & 015. 051. 092. 179. & .014. 096. 050. & .208 .104 .054 .009 & 007. 039. 089. 192. \\
\hline 150,2 & 013. 049. 096. 181 & 014. 049. 096. 181 & .03 .051 & .038.006 \\
\hline 20,3 & 011. 048. 093. 184 & . 185.093 .046 .010 & 220.109 .049$. & .008. 044. 195. \\
\hline 50,3 & 015. 051. 093. 180. & .015. 052. 180.095. & 215. 107. 050. & .093.045. 009 201 \\
\hline 150,3 & .016. 182.095 .054 & .016.053.097. & 010. 050. 102. 200. & .205. 103.047. \\
\hline 20,6 & .017. 093. 048. & 011. 048. 091. 179. & 008. 052. 109. 217. & .010. 052. 200. \\
\hline 50,6 & .017. 092. 052. & .013. 051. 180. & 218. 106. 052. & 009. 051. 099. \\
\hline 150,6 & .015. 052. 182. & .016. 053. 182. & .009. 095. 198 .095 & .011. 053. 105. \\
\hline
\end{tabular}


Table 3. Empirical size of $E_{p}, Q_{p}$ and $W_{p}$ for $p=2, n=50, M=10000$.

$$
\begin{aligned}
& E_{p} \quad Q_{p} \quad W_{p} \\
& \begin{array}{lllllllllllll}
\rho & 20 \% & 10 \% & 5 \% & 1 \% & 20 \% & 10 \% & 5 \% & 1 \% & 20 \% & 10 \% & 5 \% & 1 \%
\end{array}
\end{aligned}
$$

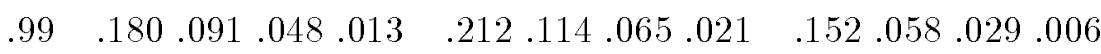

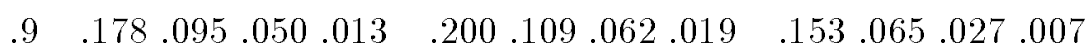

$$
\begin{aligned}
& \begin{array}{llllll}
.5 & .180 .096 .052 .013 & .182 .097 .055 .015 & .192 .090 .041 .006
\end{array} \\
& \begin{array}{llllll}
0 & .179 .092 .051 .015 & .177 .096 .050 .014 .039 .007
\end{array}
\end{aligned}
$$

\begin{tabular}{|c|c|c|c|c|c|}
\hline$\alpha$ & $\beta$ & $\begin{array}{lllllll}E_{p} & Q_{p} & M_{p} & Z_{p} & W_{p} & W_{p}^{*}\end{array}$ & $\alpha$ & $\beta$ & $\begin{array}{lllllll}E_{p} & Q_{p} & M_{p} & Z_{p} & W_{p} & W_{p}^{*}\end{array}$ \\
\hline .1 & -1 & $\begin{array}{l}.955 .139 .993 .544 .027 .912\end{array}$ & 1 & -1 & 053.054. 248.064.046. \\
\hline .1 & -.5 & .954. 145.993.573.028.906 & 1 & -.5 & 069.060.052. 238.066. 064. \\
\hline .1 & 0 & .953. 153.990.597.026.906 & 1 & 0 & 083.085.053. 251.065. 065. \\
\hline .1 & .5 & .952. 162.991. 621.025.905 & 1 & .5 & 099. \\
\hline .1 & 1 & $\begin{array}{l}.955 .179 .991 .643 .026 .911 \\
\end{array}$ & 1 & 1 & 116. 134.048 .288 .073 .043$. \\
\hline 2 & -1 & 685. 774.068 .941 .260 .039$. & 2 & -1 & 051. 041. 058. 144. 052. \\
\hline .2 & -.5 & .780.070.940. 285.035.692 & 2 & -.5 & 040.052.052. 133.060. 040. \\
\hline .2 & 0 & .788 .075 .935 .315 .032 .700 & 2 & 0 & 052.047.050. 132.059. \\
\hline .2 & .5 & .799. 084.936. 349. 029. & 2 & .5 & 058. 043. 064. 142. 051. \\
\hline .2 & 1 & .815 .095 .938 .387 .028 .731 & 2 & 1 & 065 .053.052. 146. 064 .064. \\
\hline .5 & -1 & 184. 049. 084. 543. 543. & 10 & -1 & 056.059.048.053.050. 058. \\
\hline .5 & -.5 & 197. 242.054 .542 .088 .048$. & 10 & -.5 & 053.059.047.047.055. 050. \\
\hline .5 & 0 & 218. 272.055 .552 .106 .047$. & 10 & .0 & 049.045.048.057.050. 057. \\
\hline & .5 & .311.056.577. 124.046. 251 & 10 & .5 & 051. 037. 053. 061. 051. 050. \\
\hline & 1 & 296. 369.057 .611 .144 .043$. & 10 & 1 & 052.053.053.057.053. \\
\hline
\end{tabular}

Table 4. Power at 5\% against generalized Burr-Pareto-logistic with normal marginals for $p=2, n=50, M=5000$. 

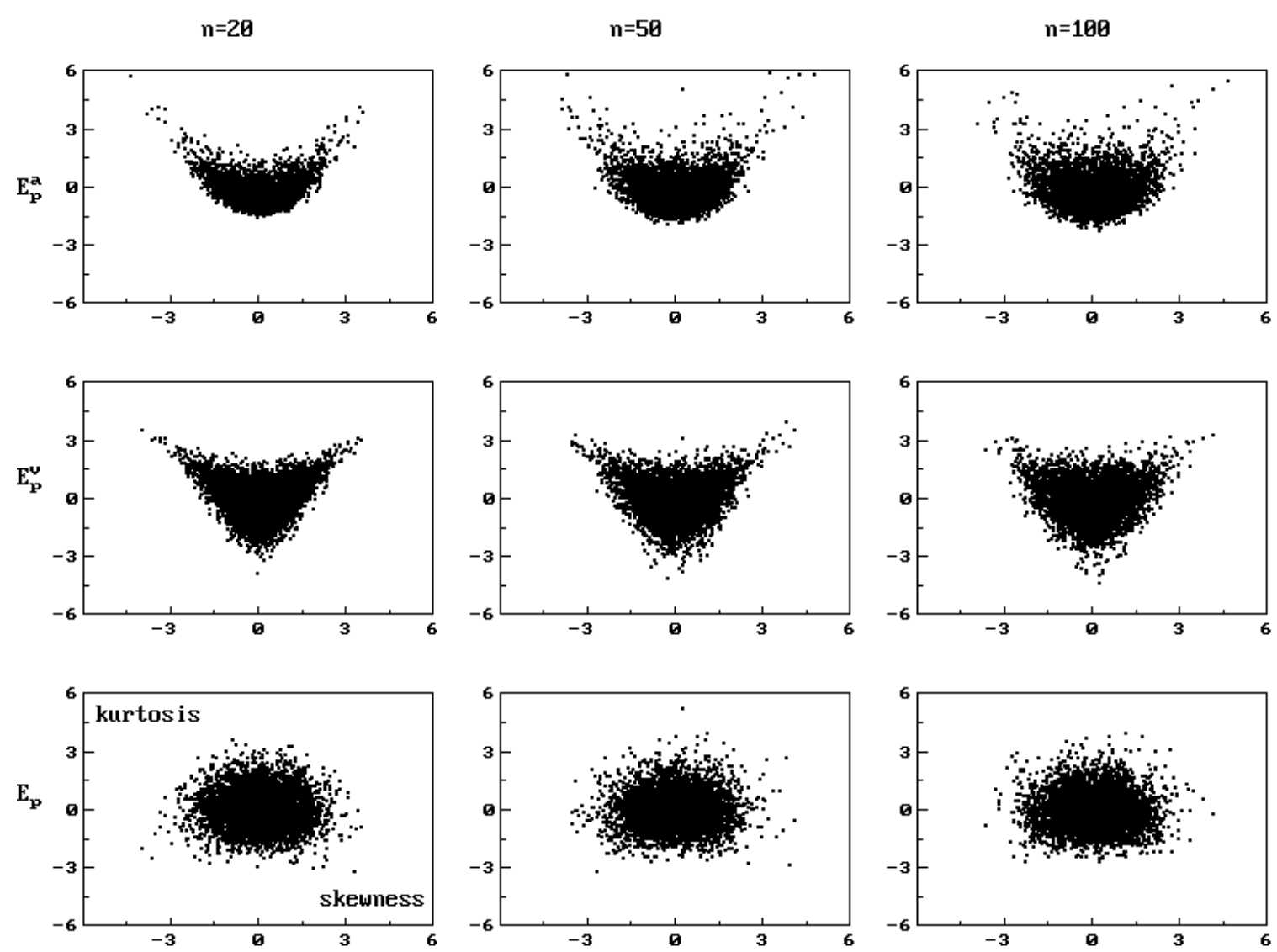

Fig. 1. Cross-plots of transformed skewness and kurtosis for 5000 samples of sizes $n=$ $20,50,100$ from the standard normal. 


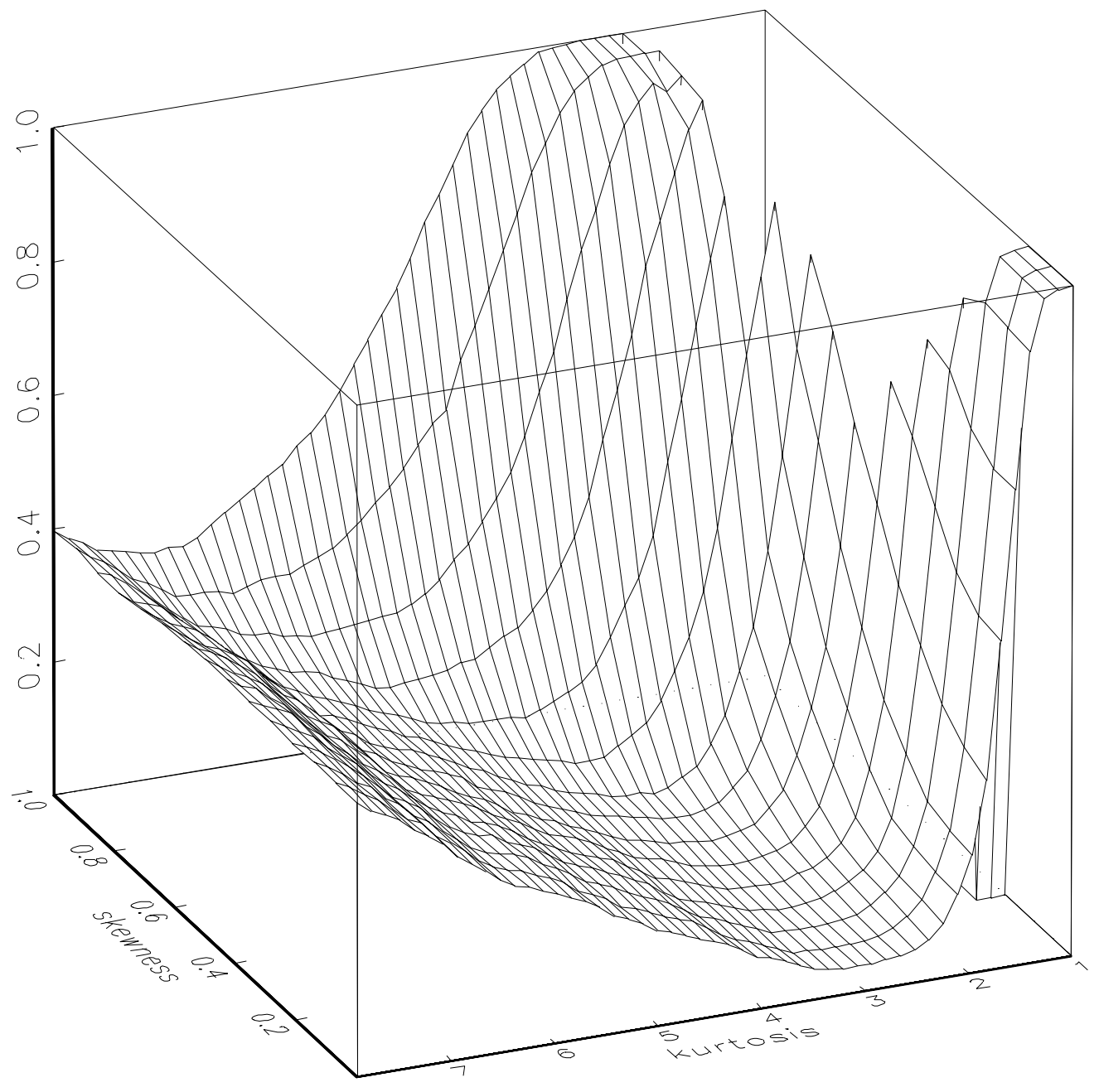

Fig. 2. P12 for $E_{p}$ : power against two standard normal marginals and the Johnson system for the third marginal. 


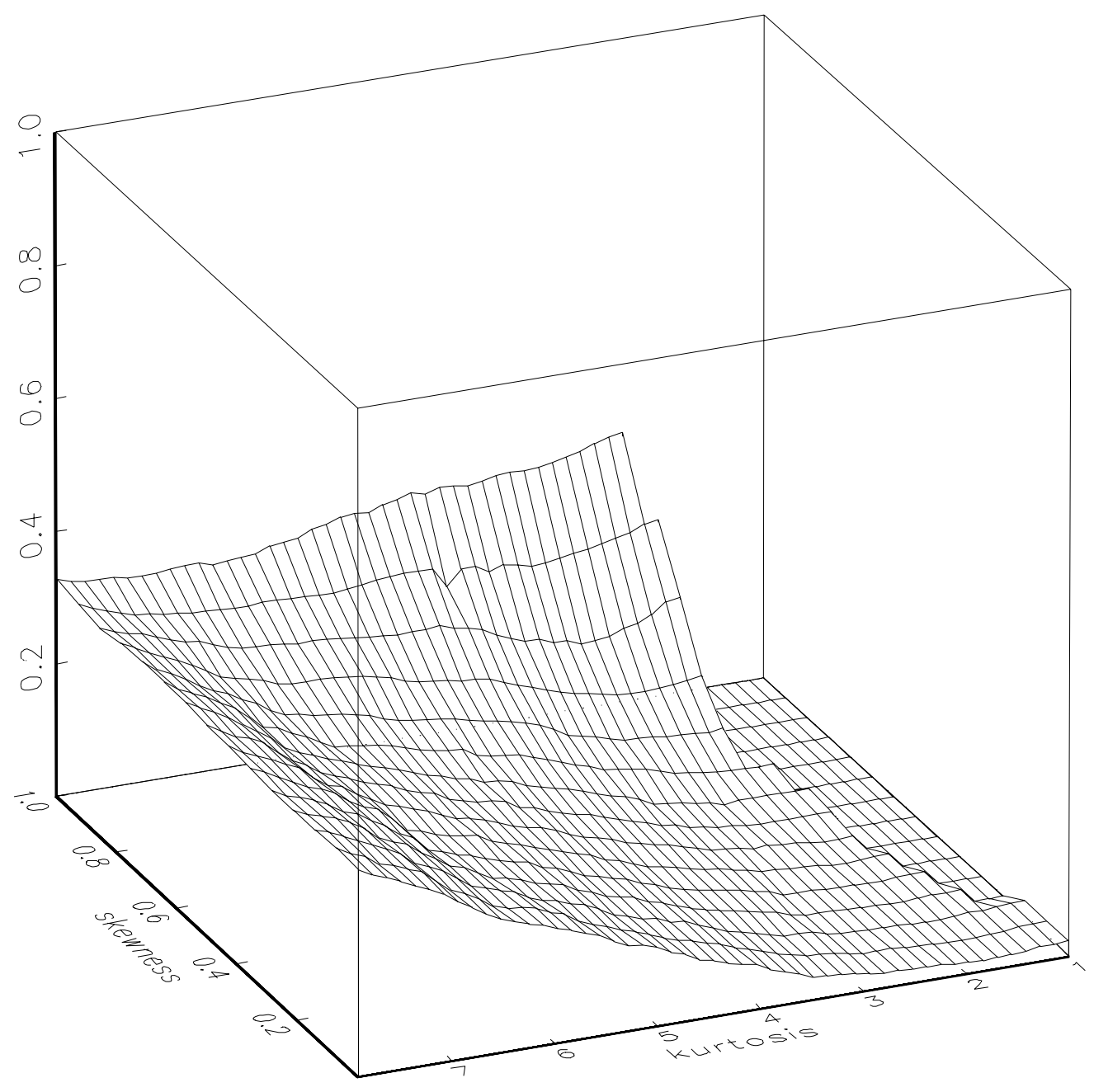

Fig. 3. P12 for $M_{p}$ : power against two standard normal marginals and the Johnson system for the third marginal. 


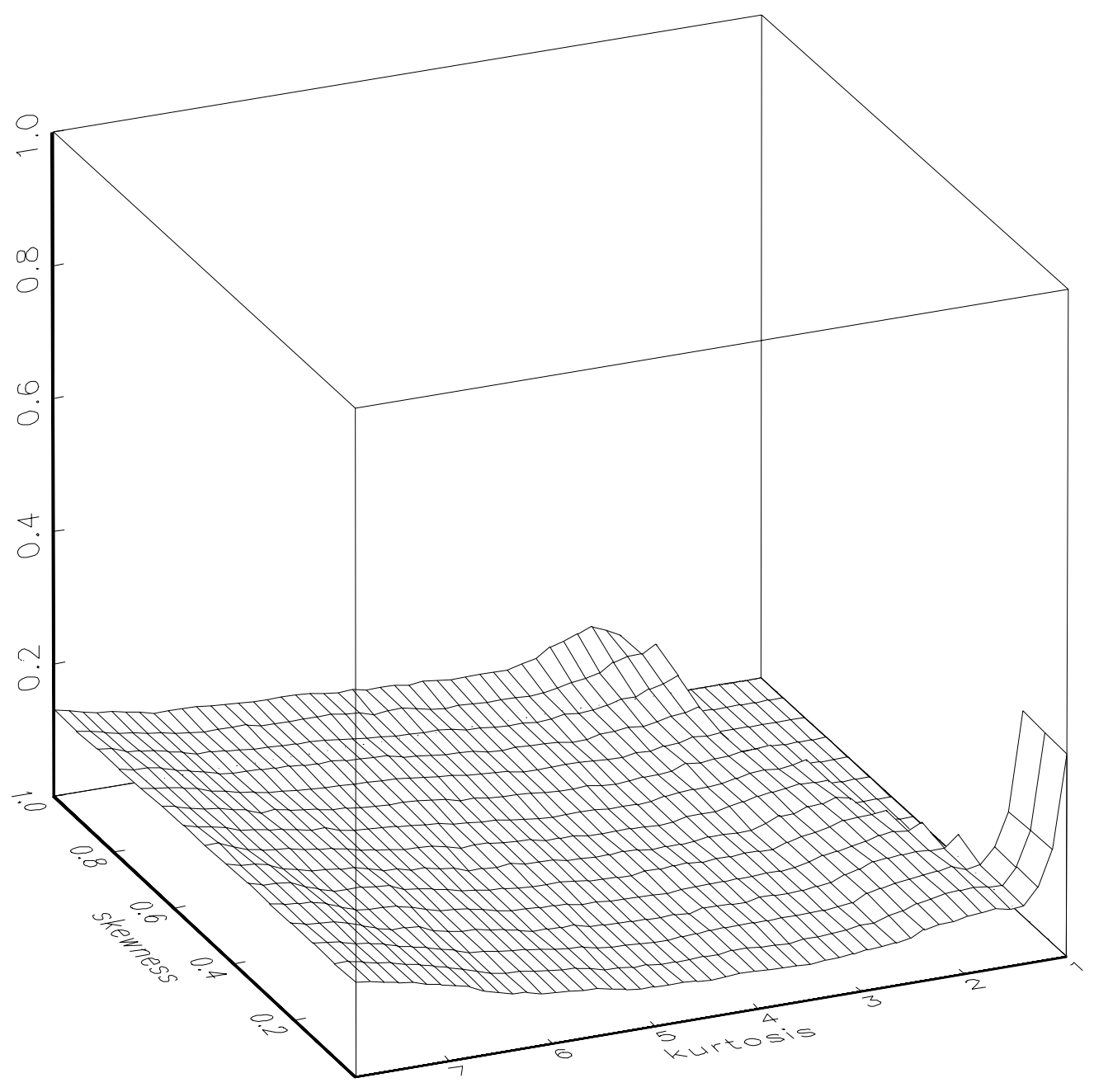

Fig. 4. P12 for $Z_{p}$ : power against two standard normal marginals and the Johnson system for the third marginal. 


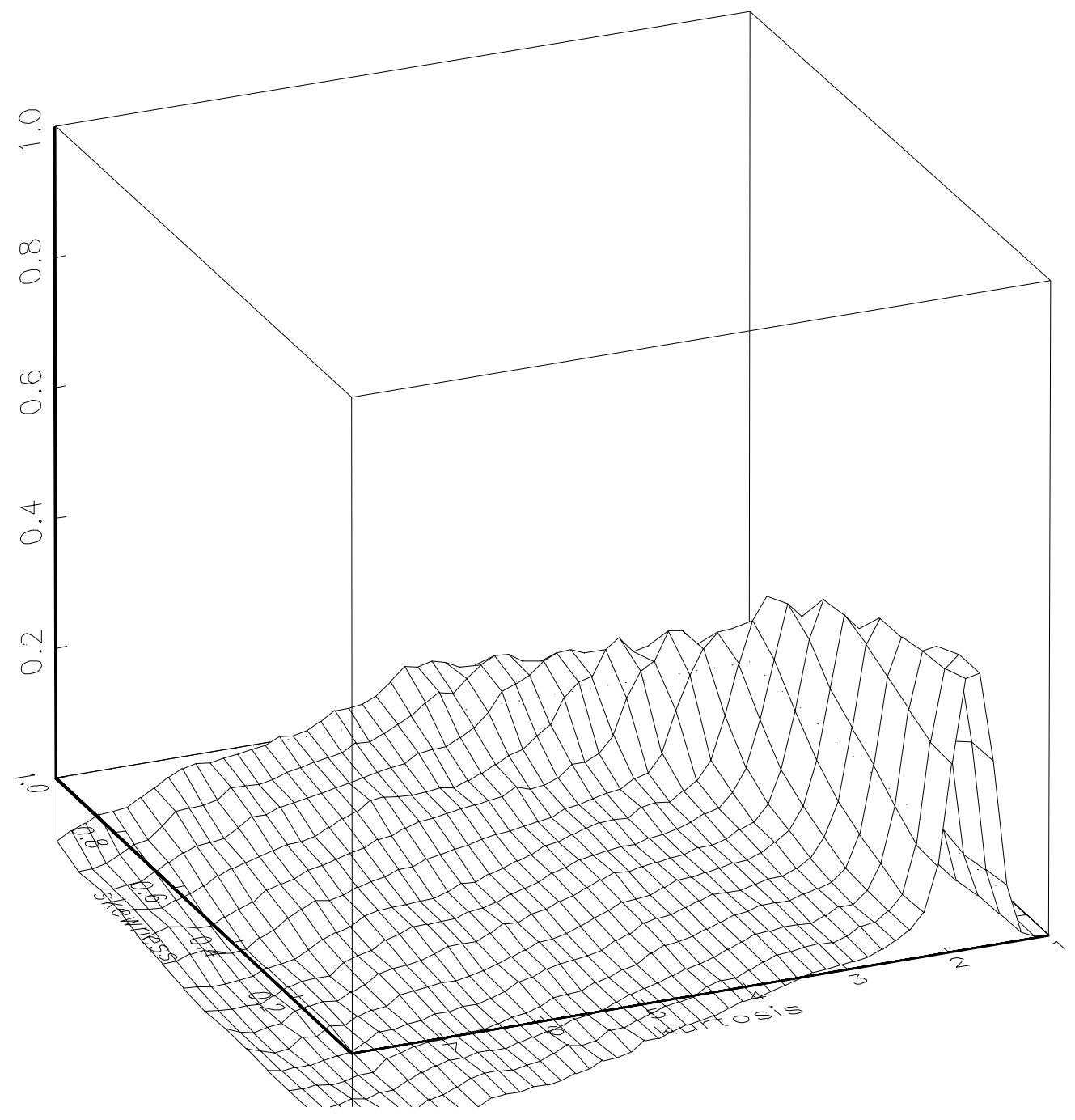

Fig. 5. P12 for $W_{p}-E_{p}$ : difference in power against two standard normal marginals and the Johnson system for the third marginal. 